\title{
Acoustic characterization of thin polymer layers for Love mode surface acoustic waveguide
}

\author{
L. El Fissi, J-M. Friedt \\ SENSEOR \\ Parc de Haute Technologie - Lot $n^{\circ} 3$ \\ 694, Avenue du Docteur Maurice Donat \\ 06250 MOUGINS France \\ lamia.elfissi@femto-st.fr \\ jmfriedt@femto-st.fr
}

\author{
S. Ballandras, L.Robert, F. Chérioux \\ FEMTO-ST, UMR CNRS 6174, UFC-ENSMM-UTBM \\ 32 Avebue de l'Observatoire \\ 25044 Besançon, France \\ ballandr@femto-st.fr
}

\begin{abstract}
Beyond the great gravimetric sensitivity provided by the polymer guiding layer of shear surface wave on quartz resulting from a low acoustic velocity, the use of photo resists provide economical means of depositing guiding layers of optimal thicknesses compared to inorganic layer deposition processes (typically PECVD deposition of silicon dioxide lasting several hours). We here analyze the evolution of the guiding layer properties, i.e. the acoustic velocity and losses, as a function of time (solvent evaporation following photo resist spin coating) and temperature (typical baking steps). The polymer films is deposited on an AT-cut quartz substrate patterned with passivated $\left(\mathrm{SiO}_{2}\right)$ inter-digital transducers $(\mathrm{Al})$ for generating 40 $\mu \mathrm{m}$-wavelength shear waves converted to a guided Love mode in a delay line configuration. We exploit experimental characterization results via a model of acoustic wave propagation to determine physical parameters of the layer. We then have determined materials coefficient such as viscosity, elastic and thermoelastic constants and density out of the velocity and insertion loss measurements.
\end{abstract}

\section{I.INTRODUCTION}

Love-mode acoustic wave sensors have demonstrated high detection sensitivity for analytes in liquid media. Beyond the qualitative identification of an analyte, the quantitative interpretation of the physical properties is a complex problem associated to the multitude of possible causes yielding changes in acoustic velocities and insertion losses. Among such phenomena are the adsorbed mass, layer permittivity, viscoelastic properties, temperature: these combined properties impose complex analysis but also provide unique opportunities for identifying such properties in thin organic films.

In order to exploit at best the rich combination of measurements associated with acoustic devices, we present the results of finite element analysis models combined with boundary conditions including viscoelastic losses applied to experimental data of organic thin films adsorbed metallic surfaces and probed by a $125 \mathrm{MHz}$ Love mode acoustic wave. A finite element/boundary element model (so-called FEA/BEM) simulating Newtonian viscous fluids accounting for viscosity frequency dependence was developed in that matter, allowing for reliable descriptions of actual transducer shapes and operation. We deduce from these models mass and viscosity properties of the layer, from which we extract meaningful quantities for further biosensor modeling including solvent content in the layer, optical index and density. The P-matrix mode characteristics are extracted from these computations to simulate the implemented devices. They consist in delay lines with inter-digital transducers protected from the presence of water which would otherwise prevent their normal operation, and a probing area on which are deposited either the probed medium or reactive layers for identification purpose. The corresponding frequency dependent phase shift and acoustic losses are introduced in the P-matrix model, allowing for an accurate prediction of insertion losses and phase sensitivity of our Love-wave delay lines.

This approach is illustrated by monitoring the evolution of a resist layer during curing, to relate the viscosity changes to the evolution of solvent concentration of the film along temperature and heat delays. In the particular configuration of our experiments, the resist layer contributes to the guiding of the Love-mode (deposited by spin-coating to obtain a $1 \mu \mathrm{m}$ thick guiding layer atop a $\mathrm{SiO}_{2}$-based waveguide), which yields specific insertion losses (IL) and delays related to the layer's properties. The tested layer consists of a Novolak-based photo-resist used in microelectronics (Shipley S1813) combined with a Propylene-Glycol-Monomethyl-EtherAcetate (PGMEA) solvent. Using the heating chuck of our tip prober allows for monitoring the evolution of the delay line transfer function for different values of temperature. We record the corresponding IL and delays allowing for tracing the layer's acoustic property changes. To calibrate the set-up, we have recorded in parallel the solvent concentration within the layer using Fourier Transformed InfraRed (FTIR) spectroscopy in order to check the coherence of our Lovemicrobalance-based experimental results with direct measurement results. We then discuss the evaluation of the equivalent viscosity of the film to deduce the solvent concentration, together with the layer's elastic and thermoelastic properties. 


\section{II.Description of the Love-Wave-BAsed Microbalance}

Our Love-wave devices consists in delay lines built on (AT,Z) cut of quartz. The wave guiding is achieved by depositing a 1 $\mu \mathrm{m}$ thick photo-resist S1813 layer atop AT-cut of quartz. The Love wave is excited and detected using IDTs composed of 50 pair of 4-finger-per-wavelength electrodes made of 200 $\mathrm{nm}$ thick evaporated Aluminum. The wavelength is fixed to $40 \mu \mathrm{m}$, yielding a frequency operation in the vicinity of 125 MHz. A $3.2 \mathrm{~mm}$ long cavity is achieved in between the two IDTs. The acoustic aperture is $3.5 \mathrm{~mm}$. The pads are covered by an adhesive tape before spin-coating the S1813 photoresist atop the wafer with the IDTs and $\mathrm{SiO}_{2}$ guiding layer. This adhesive tape is withdrawn after the coating of the photo-resist in order to open the bonding pads. Figure 1 shows a scheme of the delay line with $\mathrm{SiO}_{2}$ and $\mathrm{S} 1813$ guiding layers.

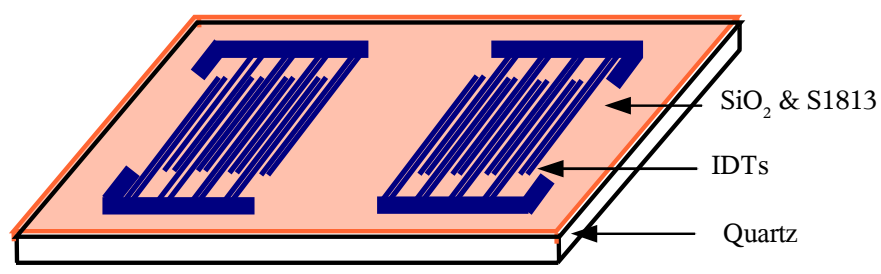

Figure 1. Scheme of the Love-wave based delay line

\section{III.Microbalance Modeling Assessement}

The transducer consists in pairs of splitted fingers to favor the device operation far from the Bragg condition. This structure however is passivated by the guiding layer, which complicates the simulation of its acousto-electric operation. We then use our periodic FEA-BEM simulation tool to account for the actual shape of one mechanical period of the IDT. In that matter, we actually consider that the overlay conforms the initial shape of the transducer, yielding the mesh of fig.2. To simply simulate the working of the transducer, we apply a phase condition corresponding to an harmonic excitation in quadrature, allowing for simply representing the actual voltage conditions applied to the transducer.

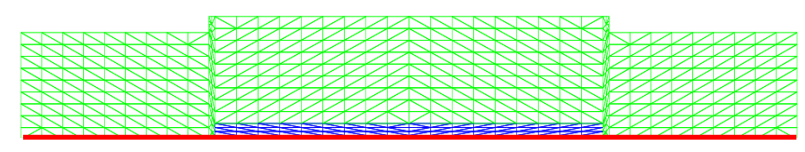

Figure 2. Mesh of one elementary electrode (one mechanical period) of a 4finger-per-wavelength IDT. The overlay is assumed conformal, i.e. it reproduces the initial shape of the transducer on the top surface. The green area is the passivation layer, the electrode is in blue and the red line indicates the radiation area.
Because far from the Bragg condition, we don't have to care about any kind of stop-band effect and we simply analyze the pole on the harmonic admittance (fig.3). This gives us access to all the the phase velocity, the coupling strength and the acoustic losses we are looking for [1].

The simulation of acoustic propagation parameters is much simpler than the one of the transduction area of the device, since plane interfaces allows for developing analytic harmonic models allowing for the derivation of surface or interface Green's functions from which the phase velocity and the leakage coefficient can be easily deduced. We then only describe here the results when have obtained by considering different concentrations of glycerol/water compound atop the silica-based Love wave guide.

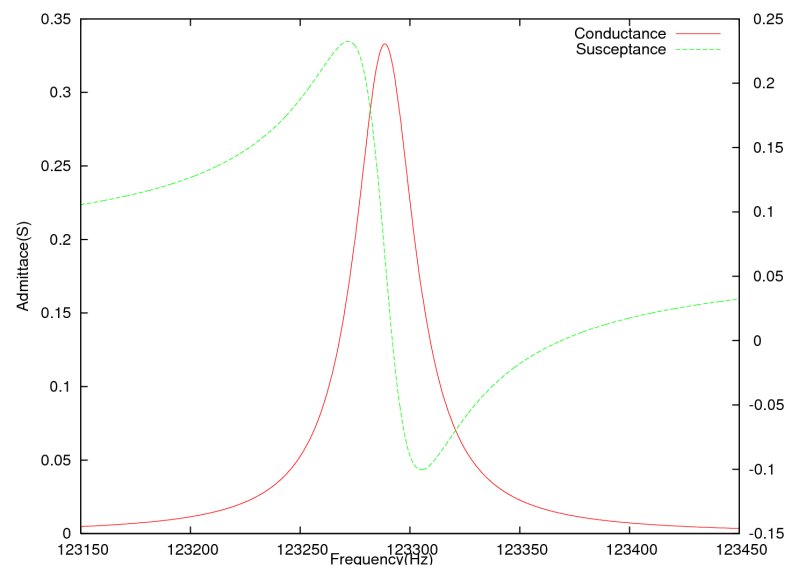

Figure 3. Pseudo-pole of the harmonic admittance for a phase quadrature excitation applied to the mesh of fig. 1

The evolution of the acoustic velocity and losses versus Glycerol concentration (fig.4) shows that the wave velocity is significantly affected by the viscosity of the liquid. This must be accounted for when measuring any deposited material atop the device, to correctly separate the different involved effects, i.e. mass loading and viscosity changes. In the present case, we also account for the role of the transducer which is submitted to the resist load, and which contributes in a non negligible matter to the actual central frequency location of the delay line. Finally, as described in [1], we combine all the extracted data in a mixed-matrix model [2] to simulate the whole Love-wave sensing delay line. Particularly, both section of the model (the two transducers and the sensing area) are coupled together in a usual matrix cascade, providing the simulation results we are looking for.

These developments have been confirmed and validated by experiments [3]. We have loaded the sensing area of our Lovewave delay lines with water/glycerol compounds, progressively increasing the glycerol concentration and monitoring the corresponding phase changes and insertion loss 
evolution of the device. We then have superimposed the predicted insertion losses to the measured ones, demonstrating the accuracy of our prediction in the viscosity range $1-20 \mathrm{cP}$. Figures 5 and 6 shows the comparison between experimental and theoretical results.

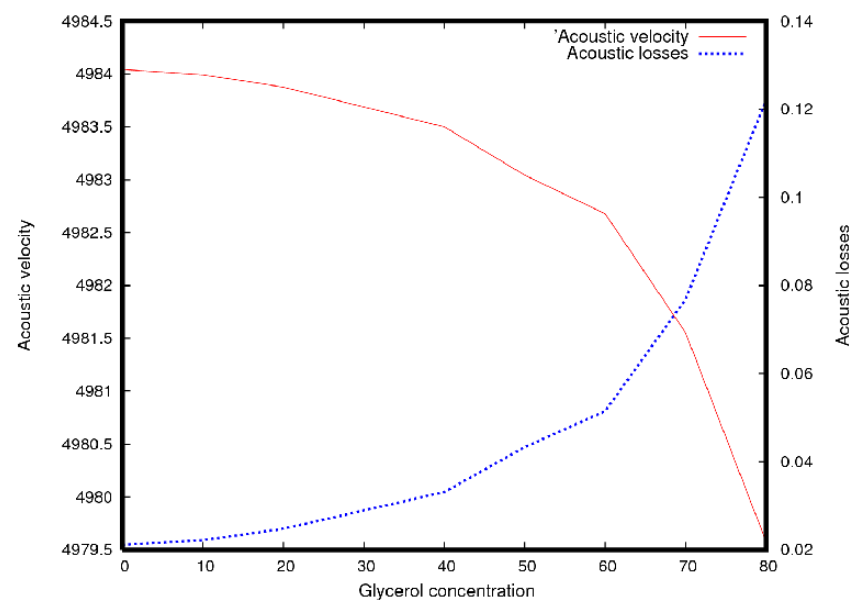

Figure 4. Phase velocity and acoustic losses versus glycerol concentration

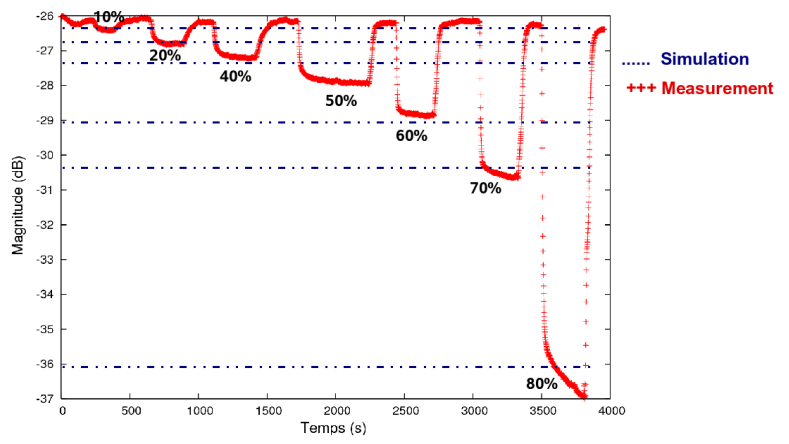

(velocity in $\mathrm{m} \cdot \mathrm{s}^{-1}$, losses in $\mathrm{dB} /$ )

Figure 5. Evolution of experimental insertion losses monitored for different concentration of glycerol, superimposition of predicted insertion loss values

For high glycerol concentrations, the stabilisation of the insertion losses is not instantaneously obtained, and relaxation phenomena seem to occur. Due to the nature of such behaviour, the proposed linear model cannot address that problem, which will require more developments to satisfyingly simulate it. However, we consider the model valid at least for small viscosity values.

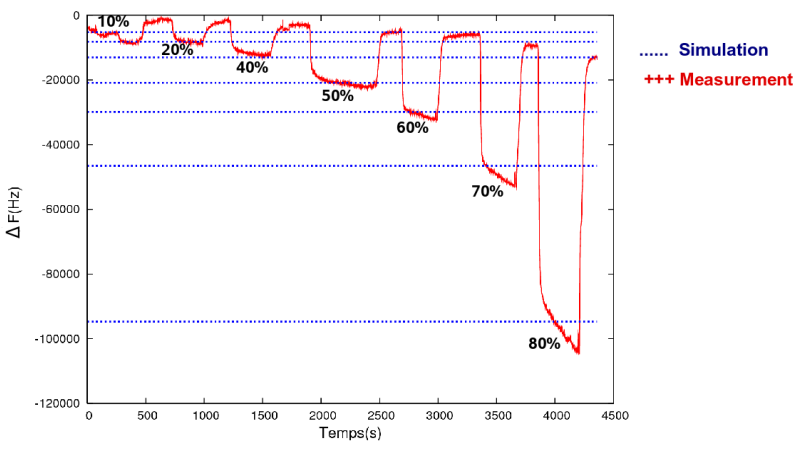

Figure 6. Evolution of experimental frequency variation monitored for different concentration of glycerol, superimposition of predicted frequency change values

\section{IV.Monitoring the Photorisest Solvent Concentration}

We now apply our experimental set-up to monitor the solvent concentration of a photo-resist layer. We then consider two experimental steps. In the first one, we evaluate the specific IL and phase velocity of the layer's when curing the resist and in a second part we recorded the solvent concentration within the layer using FTIR spectroscopy.

In order to evaluate the specific IL and phase velocity changes of the layer during curing, we record the corresponding IL and delays via a network analyzer for different values of temperature using a hot plate (Fig.7).

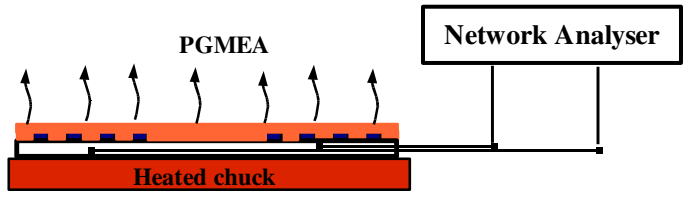

Figure 7. Scheme of the experimental set-up

As one can see in fig. 8 which present the evolution of insertion losses and frequency (phase velocity) monitored for different temperatures, the IL and phase of the microbalance are significantly affected by the state of the photresist S1813 guiding layer, i.e. mainly its solvent concentration, we intend to relate with resist physical parameters. The viscosity of the resist particularly changes due to the evaporation of the PGMEA solvent. The IL and the phase increase when increasing the temperature, which decreases the concentration of the solvent and changes the resist state from liquid to solid. We then experimentally demonstrate that the PGMEA solvent concentration affects the resist acoustic properties (as anyone would expect). We now have to correlate those changes with parameters accessible via our model. 

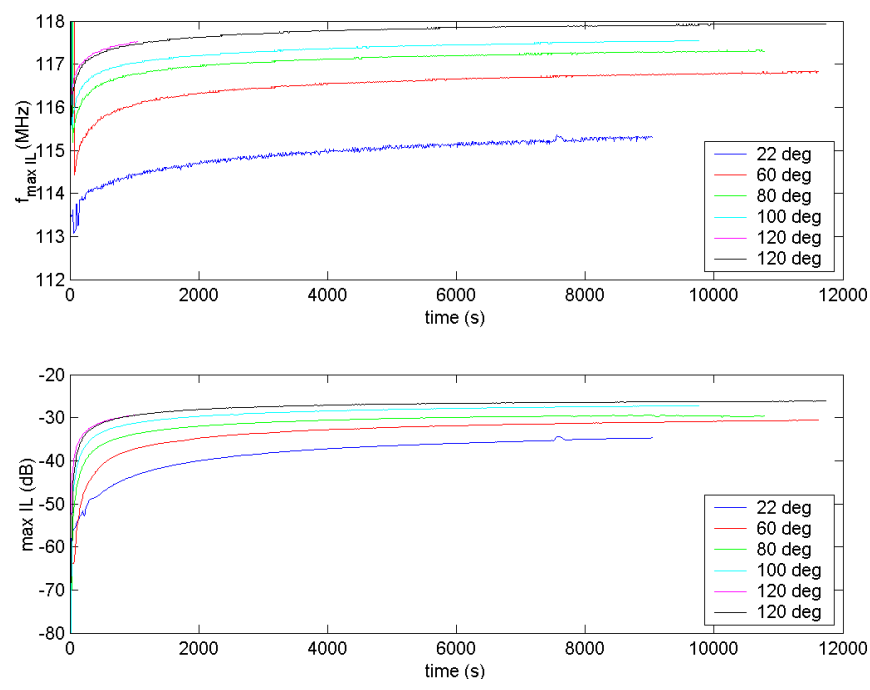

Figure 8. Evolution of experimental the delay line central frequency and insertion losses (IL) monitored for different temperatures

In that purpose, we now present the evolution of the concentration of the PGMEA solvent for different temperature using FTIR spectroscopy (Fig 9).

Infrared transmission spectra were acquired on a P-doped silicon substrate in the 400 to $4000 \mathrm{~cm}^{-1}$ range. The S1813 photoresist was spin-coated during $30 \mathrm{~s}$ at $3000 \mathrm{rpm}$, spectra were acquired within 2 minutes of spin coating, and following 5 and 15 minutes bake at 120 degrees $C$.

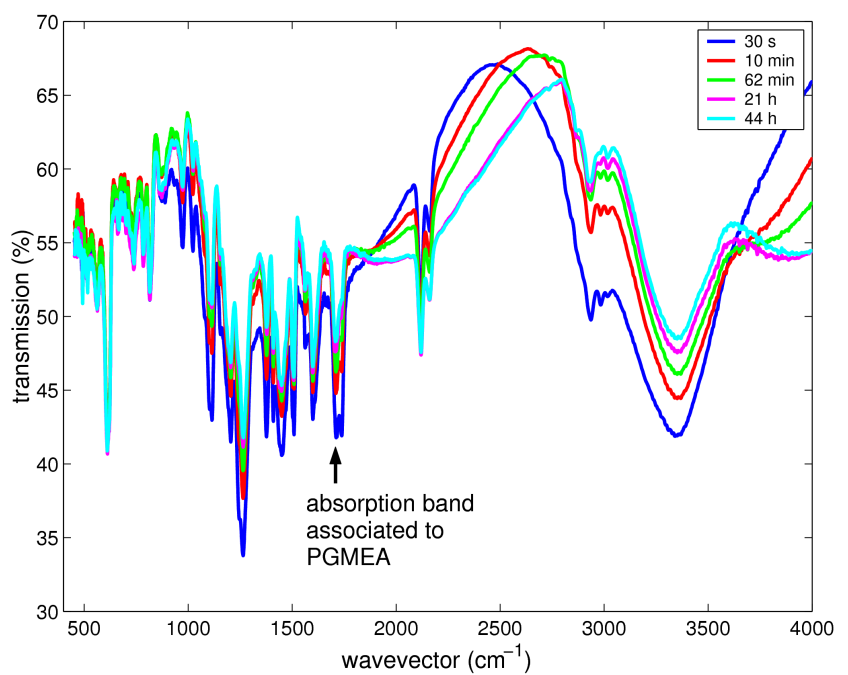

Figure 9. FTIR spectrum localizing the exploited PGMEA absorption band

The area of the absorption peak associated to PGMEA in the $1738+/-10 \mathrm{~cm}^{-1}$ is estimated to be representative of the relative weight of solvent in the layer. Since the amount of monomer in the layer will not change during the curing step, the area of the absorption peaks associated with this molecule is considered constant throughout the experiment while the solvent peak area diminishes as a function of time due to evaporation during the curing step, consistent with the constant level of the absorption peak at $810 \mathrm{~cm}^{-1}$. This absorption band is characteristic of polysubstituted aromatic rings, as expected from the cresol groups present in the monomer [4].

We finally shows the evolution of the PGMEA absorption for various temperature, obtained as explained above.

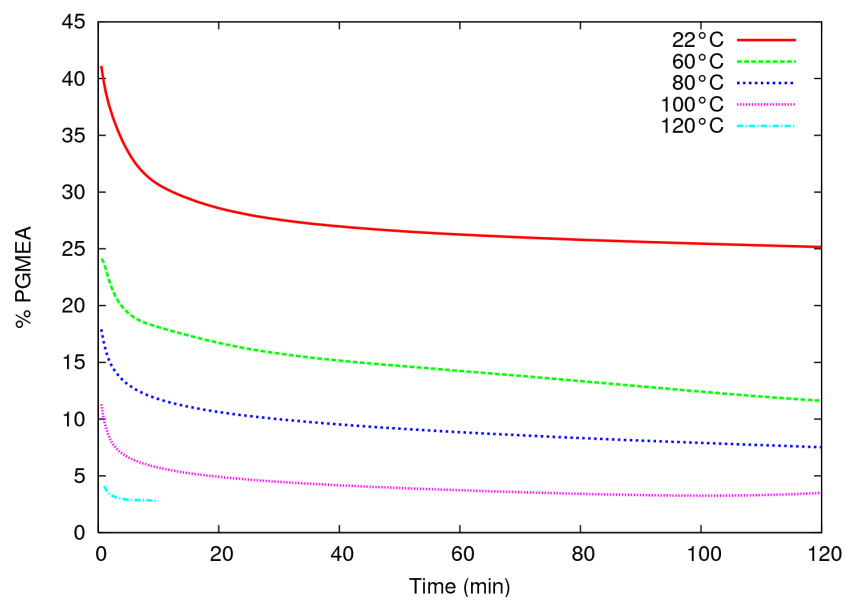

Fig. 10 Evolution of solvent concentration in the resist for different temperature using Infra-Red spectroscopy

Comparing figs 8 and 10 allows for pointing out correlations between information extracted using the microbalance and FTIR measurements.

\section{Theoretical Results}

Before we start this part, we present here the evolution of experimental insertion losses and frequency monitored for different temperatures for the SAW device without S1813 photo-resist ( Quartz $+\mathrm{SiO}_{2}$ ).

One can see in fig.11 a\&b that the SAW device is almost insensitive to thermal changes and particularly presents a quadractic frequency-temperature behaviour, with a turnover temperature close to ambiant conditions. This means we can consider that only the modifications of the photo-resist layer account in the phase and IL thermal variations observed in figure 8 . In conclusion, we can use the Love-mode device to characterize the S1813 photo-resist and to extract the physical coefficients of this resist, providing we are capable to develop a reliable model of the observed phenomena.

We first assume that the photo-resist layer behaves like a viscous fluid $\left(\mathrm{C}_{66}=0\right)$ to decouple viscosity and elasticity dependent variations. Using the Green's function of the sensing area, we have determined the viscosity coefficient by identifying the acoustic losses in the film for each temperature. We then obtained a temperature dependent 
viscosity characterizing the film changes once cured.

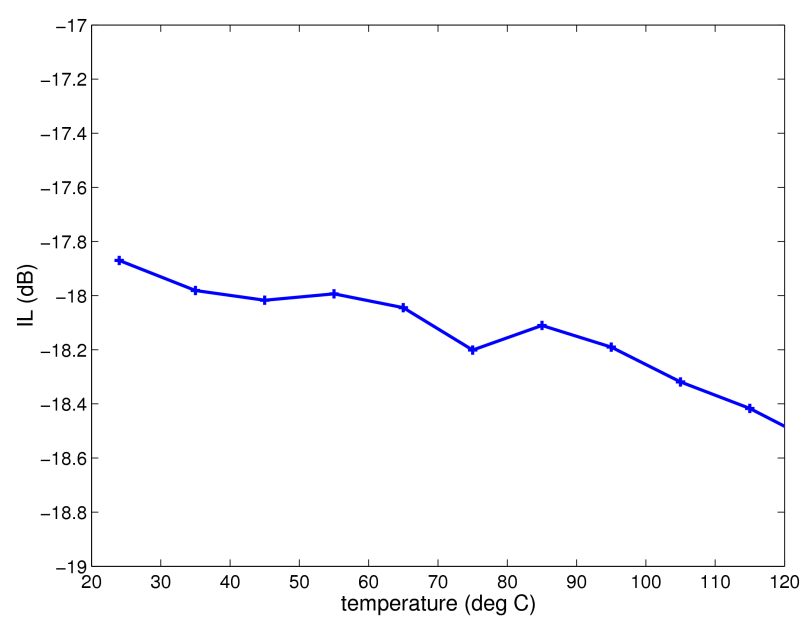

(a)

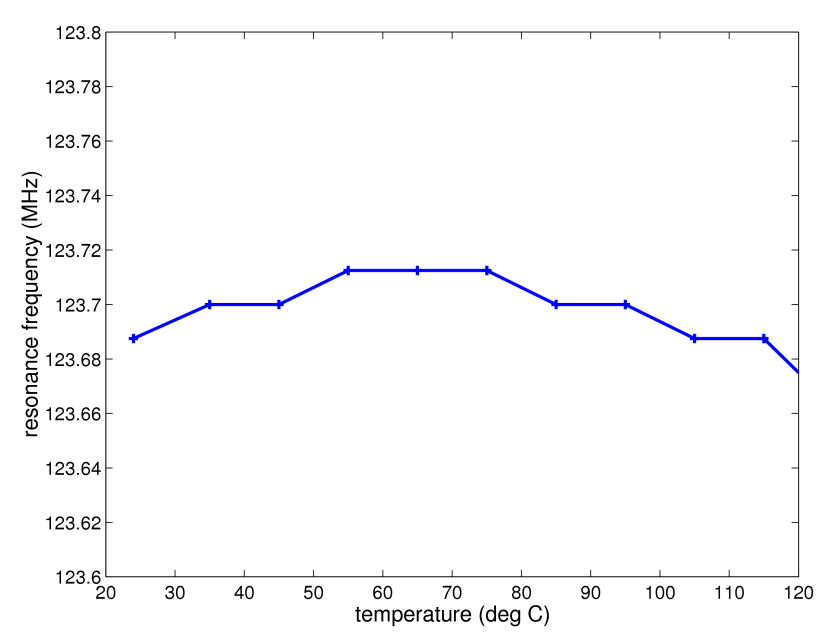

(b)

Figure 11. Evolution of experimental Insertion Losses (a) \& Frequency (b) monitored for different temperatures without the photo-resist

During this stage, we note that the variations of the frequency shift induced by the viscosity are negligible compared with the measurements of fig. 8 , and the variations of the layer thickness and density have no impact on the propagation velocity. We then have decided to modify the model and to assume that the resist behaves like a "quasi-fluid" (elastic) material $\left(\mathrm{C}_{66} \neq 0\right)$. The Poisson's ratio however remains very close to 0.5 , justifying the term quasi-fluid used above. We then have to identify a guessed elastic constant which must be inserted in our model. We find in [5] that the Young modulus of related materials should be smaller than a couple of Gpa, ranging from 3 to 0.5 or even less. To avoid too numerous computations, we have fixed it to $1 \mathrm{Gpa}$ and then deduced the shear elastic constant $\mathrm{C}_{66}$. Also the mass density should be reasonably close to the one of water, as it is the case for many organic polymers [5]. We then consider $1200 \mathrm{~kg} \cdot \mathrm{m}^{-3}$ as a reasonable value for $\rho$. Since experimental measurements were performed at various temperature, we finally have to consider the thermal expansion of the resist, which should range between 100 and $150 \mathrm{ppm} / \mathrm{K}$ according to [5], but also the thermoelastic changes which is the less known parameter for polymers. We keep the previously determined temperaturedependent viscosity coefficients and we thenextract the temperature dependent shear elastic constant.

For each temperature and using these formulas we can determine:

- the thickness of the layer: $e=e_{0}\left(1+\left(T-T_{0}\right) \alpha_{11}\right)$

$$
\alpha_{11}=150 \mathrm{ppm} / \mathrm{K} \quad T_{0}=25 \mathrm{C}^{\circ}
$$

- the mass density: $\quad \rho(T)=\rho_{0} / V_{m e}(T)$

$$
V_{m e} \text { : thermal expansion coefficient of the elementary mesh }
$$

With the above formulas and using the Green's function of the structure, we can determine the shear elastic constant for each temperature.

$$
C_{i j}(T)=C_{i j_{0}}\left(1+\left(1 / C_{i j_{0}}\right) *\left(d C_{i j} / d T\right) *\left(T-T_{0}\right)\right)
$$

$d C_{i j} / d T$ : Effective temperature derivative of the shear constant

These parameters are dominant in the variation of the frequency.

We now present the results of this section, and first the extraction of the temperature dependent viscosity parameters. Using the Williams-Landel-Ferry model in that matter, because of its known appropriateness to polymer, we states the temperature dependent viscosity as follows:

$$
\mu(T)=\mu_{0} \cdot \exp \left(-C_{1}\left(T-T_{r}\right) /\left(C_{2}+T-T_{r}\right)\right)
$$

where $\mu_{0}$ is the viscosity at $T_{r}$ the reference temperature, $C_{1}$ and $\mathrm{C}_{2}$ are empiric parameters. When $\mathrm{T}_{\mathrm{r}}=\mathrm{T}_{\mathrm{g}}$ the glass transition temperature, we should consider $\mathrm{C}_{1}=17.44$ and $\mathrm{C}_{2}=51.6$ valid for numerous polymers. In our case, a parameter fit yields the following constant values: $\mathrm{C}_{1}=-8,2$ and $\mathrm{C}_{2}=-179,4 \mathrm{~K}$. $\mathrm{T}_{\mathrm{g}}$ is close to $120^{\circ} \mathrm{C}$ for the consider photoresist. As shown in Fig.12, the model matches very well the experimental set of data.

We then focus on the evolution of elastic constant for different temperatures (Fig .13). Still using the Green's function formalism, we were able to compute the equivalent elastic constant for each temperature, yielding the dependnece reported in Fig.13. 
Using a linear fit of this curve, we deduce the effective temperature derivative of the shear elastic coefficient as follows:

$$
d C_{i j} / d T=3,110^{-5} \mathrm{GPa} / \mathrm{K}
$$

\section{Conclusion}

In this paper, we have briefly presented in the first part our Love-wave based set-up and we have shown that it can be used to quantitatively evaluate viscous properties of liquids deposited atop the sensing aera. The set-up was used to characterize the acoustic behavior of a S1813 thin photo resist layer participating to the guiding of the Love-wave and submitted to curing effect. The developed model was used to extract physical parameter and coefficients of the layer.

We particularly have shown that the property changes of the photo resist layer result primarily in the evolution of the viscosity (yielding IL changes) and the elastic behavior (yielding the main frequency changes observed) while layer thickness and density are of course affected by curing, but with a rate that does allow tyo observ their actual influence on the device characteristics.

This approach sounds to be applicable to any type of polymer film thinned by spin coating and could be extended to Rayleigh-wave-based guide to identify the other elastic constants and viscosity parameters (longitudinal).

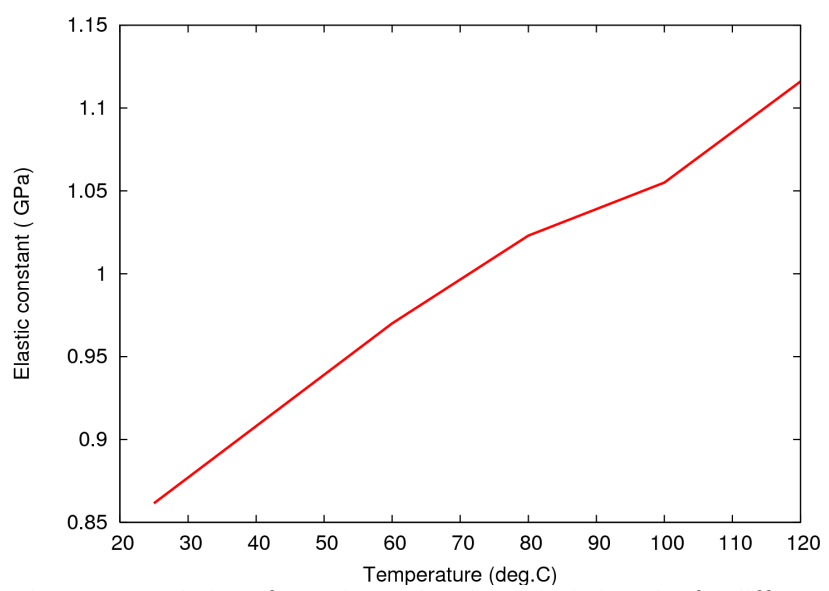

Figure 12. Evolution of experimental \& theoretical viscosity for different temperatures

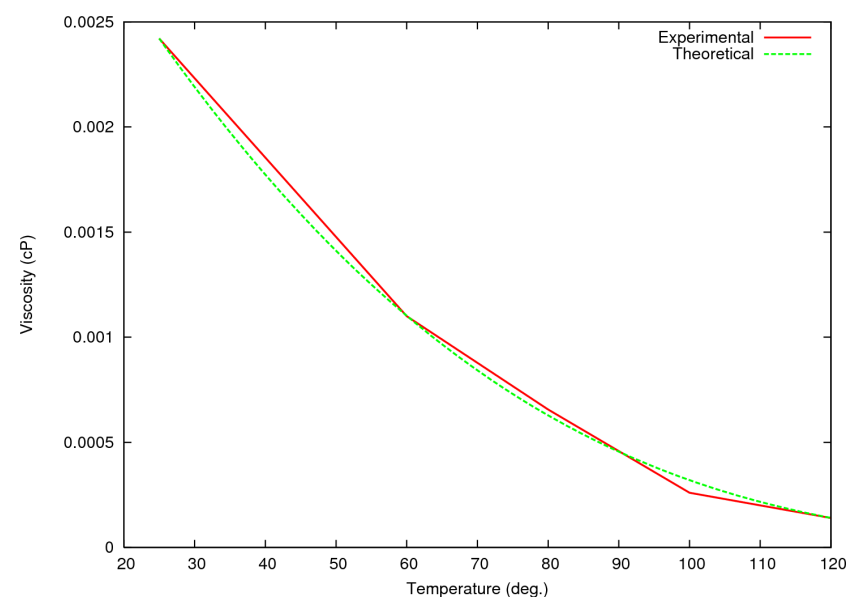

Figure 13. Evolution of elastic constant for different temperatures

Acknowledgment

The authors would like to thank Gerhard Heider (SENSeOR's CEO) for his help in promoting this work.

\section{REFERENCES}

[1] P. Ventura, J.M. Hodé, J. Desbois, M. Solal, "Combined FEM and Green's function analysis of periodic SAW structure, application to the calculation of reflection and scattering parameters", IEEE Trans. on UFFC, Vol. 48, $\mathrm{N}^{\circ}$ 5, pp.1259-1274, 2001

[2] J.M. Hodé, J. Desbois, P. Dufilié, M. Solal, P. Ventura, "SPUDT-based filters : Design, Principles and Optimization", Proc. of the IEEE Ultrasonics Symposium, pp. 39-47, 1995

[3] L. ElFissi, J.-M. Friedt, S. Ballandras, "Modeling the Rf Acoustic Behavior of Love-Wave Sensors Loaded with Organic Layers", Proc. of the IEEE Ultrasonics Symposium, New-York, pp. 484-487, 2007

[4] Reiko Saito, Yuji Ichinohe, Masahiro Kudo TOF-SIMS analysis of chemical state changes in cresol-novolak photoresist surface caused by $\mathrm{O} 2$ plasma downstream, Applied Surface Science, Volume 142, Issues 1-4, April 1999, Pages 460-464

[5] http://web.mit.edu/course/3/3.11/www/modules/props.pdf 